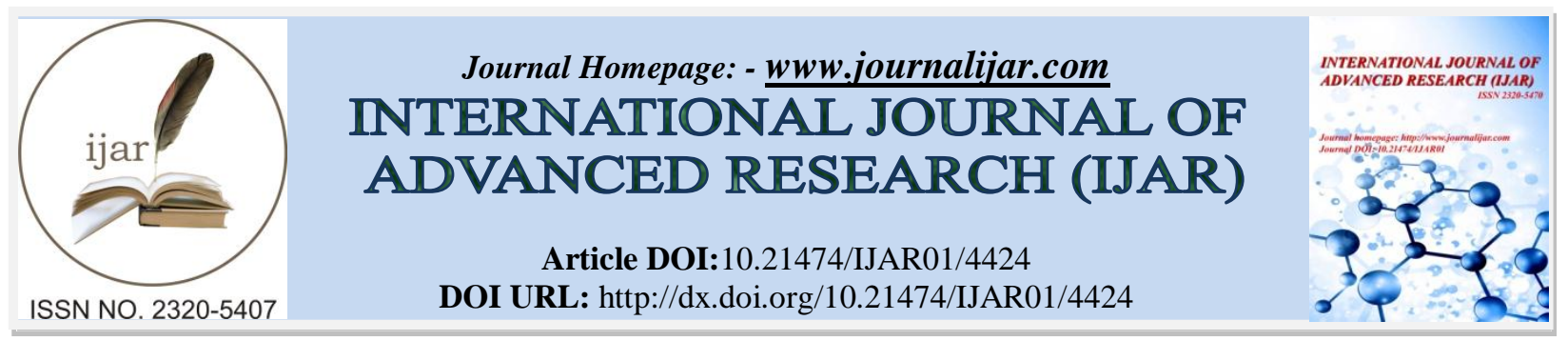

RESEARCH ARTICLE

\title{
SUIVI DE LA QUALITE PHYSICOCHIMIQUE DE L'EAU DU RIVE DROITE DU FLEUVE SENEGAL: CAS DU CAPTAGE DE LA PRISE D'EAU A KEURMACEN ALIMENTANT EN EAU POTABLE LA VILLE DE NOUAKCHOTT. MONITORING OF WATER PHYSICO CHEMICAL QUALITY OF THE SENEGAL RIVER: THE CASE OF CAPTURE OF KEURMACEN SUPPLYING DRINKING WATER OF THE SITY OF NOUAKCHOTT.
}

\section{Brahim Ould Ahmed Ould Dick ${ }^{1,2,3},{ }^{*}$ Hassan Chaair ${ }^{1}$, Khalid Digua ${ }^{1}$, Mohamed Sid'ahmed Kankou $^{2}$ and et Mohamed El Kory Ould Cheikh ${ }^{3}$.}

1. Laboratoire de Génie des Procédés et Environnement Faculté des Sciences et Techniques Mohammedia, Université Hassan II, Maroc.

2. Unité de recherche Eau, Pollution et de l'environnement, FST, Université de Nouakchott AL-Asseriya, Mauritanie.

3. Laboratoire de chimie de l'eau à l'Institut National des Recherches en Santé Publique (INRSP), Mauritanie.

\section{Manuscript Info}

\section{Manuscript History}

Received: 09 April 2017

Final Accepted: 11 May 2017

Published: June 2017

Key words:-

Fleuve du Sénégal, Nouakchott, Eau, qualité d'eau, Santé, Caractéristiques physico-chimiques

\section{Abstract}

Le présent travail concerne l'étude de la pollution physico-chimique des eaux du fleuve Sénégal. L'intérêt de ces eaux réside essentiellement dans les usages domestique et agricole qu'elles assurent; aussi il convient de suivre leur état de pollution. Dans la vallée, les activités urbaines, industrielles et agricoles s'intensifient d'une année à l'autre et par conséquent, les polluants affectent la qualité du milieu aquatique et sa composition faunistique. Cette étude effectuée au niveau de la prise d'eau à Keur macèn alimentant en eau potable la ville de Nouakchott, pour évaluer qualitativement et quantitativement la qualité des eaux du fleuve Sénégal. Un suivi mensuel sur une période de 9 mois (du janvier à septembre 2016) a été réalisé.

L'objectif de notre travail est de quantifier la pollution des eaux du fleuve Sénégal utilisé pour la production d'eau potable pour la ville de Nouakchott. Pour évaluer la qualité des eaux de rivière, plusieurs paramètres sont suivis telle que la température ; le $\mathrm{pH}$; la conductivité ; la turbidité ; le titre hydrotimétrique ; le titre alcalimétrique ; les nitrates ; les nitrites ; oxygène dissous, MES ; les chlorures, etc.

Les résultats de la présente étude ont montré que la concentration de la quasi-totalité des paramètres physico-chimiques répond aux normes en vigueur avec une turbidité variante de 21.3NTU en Mai jusqu'à 330NTU en Aout, ce qui en relation avec les MES qui présente une valeur maximal de $275 \mathrm{mg} / \mathrm{L}$ durant le mois d'Aout et les valeurs du MO minimal de $0.7 \mathrm{mg} / \mathrm{L}$ en Janvier et une valeur maximale en Septembre de $5.5 \mathrm{mg} / \mathrm{L}$ et oxygène dissous minimal de $3.77 \mathrm{mgd}^{\prime} \mathrm{O}_{2} / \mathrm{L}$ en Septembre et une valeur maximale au mois d'Avril de $6.81 \mathrm{mg}$ d' $\mathrm{O}_{2} / \mathrm{L}$

Corresponding Author:-Brahim Ould Ahmed Ould Dick. 


\section{Introduction:-}

Dans la plupart des pays en voie de développement, les eaux de surface subissent une dégradation notable de leur qualité. En effet, les eaux usées des villes sont évacuées d'une façon précaire et sans traitement préalable dans les eaux de surface. Ces rejets contiennent des concentrations importantes de micropolluants organiques, de nutriments et des éléments traces métalliques souvent à des doses toxiques, et entrainent ainsi une dégradation considérable de la qualité des eaux des Oueds [1]. La pollution due aux pratiques agricoles a été aggravée, durant ces dernières années par l'évolution de l'ensemble des systèmes de production agricole augmentant notamment leur vulnérabilité [2].

En Mauritanie, les ressources en eau de surface sont essentiellement constituées dans le fleuve Sénégal, qui réapprovisionne quatre pays à savoir : la Guinée, le Mali, la Mauritanie et le Sénégal. Ils drainent un bassin versant de $340000 \mathrm{~km}^{2}$ qui est alimenté par trois affluents principaux : le Bafing, le Bakoye et la Falame qui prennent tous les trois leurs sources dans le massif du Fouta Djalon en Guinée.

A ce jour, l'état des rivières et des estuaires africains a été très peu étudiée, alors qu'ils sont le lieu d'une grande évolution démographique et reçoivent volume croissant de déchets, en particulier des eaux usées domestiques. Le seul grand fleuve de la côte nord-ouest de l'Afrique est du fleuve Sénégal. Le nombre d'habitants sur le bassin du fleuve Sénégal est d'environ 12 millions [3].

Le potentiel en eau de la Mauritanie, est inégalement réparti dans le pays. Il est très important dans la zone du bassin côtier, et probablement dans la zone de Dhar de Nema. Cependant, il est moins important voir critique dans le reste du pays où se situe la grande partie de cités et les centres urbains secondaires. L'importance stratégique des eaux de surface exige une surveillance périodique constante de leur qualité physicochimique et bactériologique. La pollution des eaux de surface pourrait engendrer diverses maladies telles que : choléra, typhoïde, hépatites, bilharziose et intoxications chimiques [2].

Le diagnostic des eaux du fleuve Sénégal permettra de caractériser les eaux entre autre et d'obtenir par la suite un aperçu de l'état de la qualité des différentes masses d'eau du bassin utilisé pour la production d'eau potable pour la ville de Nouakchott. Ceci permet de renforcer significativement les connaissances sur la qualité physicochimique des eaux pour améliorer la qualité des eaux potable alimentant la ville de Nouakchott en respectant les normes de potabilisation

Avec la croissance démographique et la sécheresse que connaît la Mauritanie ainsi que les changements climatiques et le développement industriel et agricole, le contrôle et la surveillance de la qualité des eaux de surface suscitent un intérêt particulier.

Pour évaluer la qualité des eaux de surface, trois types de critères de qualité ont été élaborée:-

A. Des critères de qualité descriptifs qui fournissent des règles générales pour protéger les eaux de toute dégradation grossière.

B. Des critères de qualité chimiques qui spécifient les seuils sans effet pour les contaminants considérés un à un.

C. Des critères de qualité relatifs à la toxicité globale qui permettent d'évaluer la toxicité d'un mélange de substance.

L'objectif principal de cette étude est la préservation de la santé de la population mauritanienne en évaluant la qualité de l'eau de boisson distribuée.

Cette étude évaluer la nature, le degré et l'origine de la pollution qui touche les eaux de surface du fleuve Sénégal ainsi que les effets de cette pollution sur la production d'eau potable.

\section{Matériels and Méthodes:-}

\section{Présentation de la zone d'étude:-}

La zone d'étude choisie appartient au delta du fleuve Sénégal (prise d'eau à Keur macèn) qui se situe dans la partie sud-ouest du Trarza. Elle est limitée à l'Est par le lac de R'kiz, au nord par le cordon dunaire qui borde la 
vallée du fleuve, à l'ouest par le barrage de Diama qui se situe juste à l'embouchure du fleuve Sénégal et l'océan Atlantique et au sud par le courant du fleuve.

Le fleuve Sénégal s'écoule dans le sens est-ouest sur une longueur de $1800 \mathrm{~km}$ de drainage jusqu'à l'embouchure de Diama. Il naît de la rencontre du Bafing, le fleuve "noir", descendu du Fouta-Djalon à 800 mètres d'altitude, et du Bakoye, le fleuve "blanc" qui prend sa source sur le plate au Mandingue à 300 et 500 mètres d'altitude. Son principal affluent est la Falémé, qui draine toute la partie Est du Sénégal. Le fleuve Sénégal approvisionne quatre pays : la Guinée, le Mali, la Mauritanie et le Sénégal [4]. La population qui y vit représente $16 \%$ de celle des trois pays riverains (la moitié au Sénégal, $5 \%$ au Mali et le reste en Mauritanie) [5].

\section{Le bassin est divisé en trois grandes régions:-}

- Le haut bassin qui est montagneux ;

- la vallée qui est connue depuis longtemps comme une zone agropastorale des pays riverains et - le delta qui est une région de grande diversité biologique et qui abrite plusieurs zones humides [4].

- Le delta du fleuve Sénégal se trouve en zone sub-saharienne à faible pluviométrie (30 $\mathrm{mm}$ en moyenne), son climat est de type sahélo-saharien et comprend généralement deux saisons dans l'année :

$>$ la saison des pluies de juin à septembre ;

$>$ la saison sèche d'octobre à juin.

De nos jours, il est difficile d'établir une série chronologique continue de la pluviométrie du bas delta, malgré l'existence de plusieurs stations météorologiques (Rosso, R'kiz, Diama, etc...), ce qui rend difficile l'interprétation des données climatiques. Cependant, la moyenne pluviométrique retenue officiellement est de $300 \mathrm{~mm}$ par an pour les dix dernières années [4].

Les moyennes annuelles de précipitations enregistrées entre 1981 et 2011 sont de l'ordre de $189 \mathrm{~mm}$ à Rosso. Les températures de l'air généralement élevées, sont caractérisées par de larges fluctuations entre le jour et la nuit. Ce contraste est d'autant plus important à mesure que l'on rentre dans le continent (gradient nord-est bien marque). C'est ainsi que la température moyenne varie de $25^{\circ} \mathrm{C}$ a proximité du littoral a $30^{\circ} \mathrm{C}$ dans le continent. La figure 1 montre la prise d'eau Aftout Essahili.

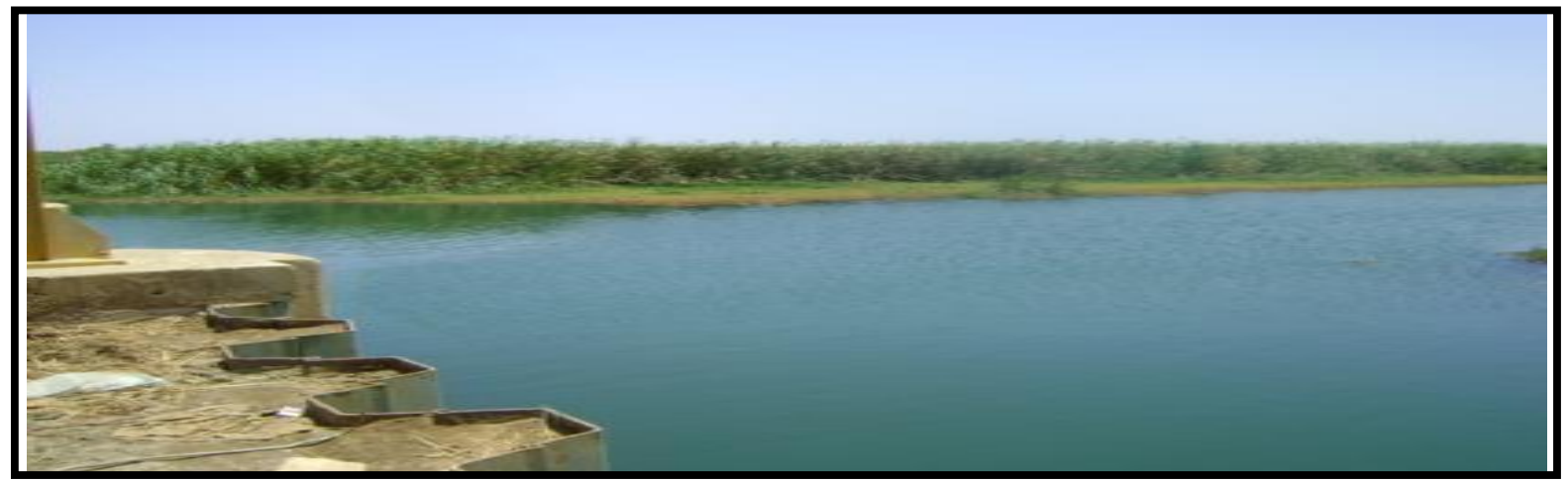

Figure 1:- la prise d'eau Aftout ES SAHL à keur mecén [6].

\section{Lieux des prélèvements et échantillonnages :-}

Les prélèvements ont été effectués au niveau de la prise d'eau Aftout ES SAHLI à environ $6 \mathrm{~km}$ de Keur mecèn dans des flacons en verre borosilicaté bouchés, rincées à l'eau distillée et en fin séchés pour être utilisés. Un suivi mensuel sur une période de 9mois, du janvier au septembre 2016) ont été réalisés.

L'échantillonnage mensuel des eaux de surface, à risque de contamination agricole, permet de caractériser l'état physico-chimique de ces eaux au fur et à mesure du déroulement des saisons et en fonction des cycles de culture.

\section{Les Analyses physico-chimiques:-}

Les paramètres physicochimiques ont été analysés selon la méthode norme AFNOR 1999 [7]. Les mesures de la température, du pH, de la turbidité et de la conductivité électrique ont été réalisées in situ. 
La température et le $\mathrm{pH}$ des eaux ont été mesurés à l'aide d'un pH-mètre (Hanna 205). La mesure de la turbidité a été réalisée par un turbidimètre de type (TU 2100P). La conductivité a été mesurée par un conductimètre de type (H sension 7). L'oxygène dissous a été mesuré par un oxymétrie de type (sension $\mathrm{TM}+\mathrm{DO6})$.

Le $\mathrm{TH}$, le TAC et les matières organiques ont été mesurés par la méthode volumétrique et le reste des paramètres (NO3,NO2, MES, Chlorure, et $\mathrm{O} 2$ ) ont été analysés par la méthode spectroscopie à l'aide d'un spectrophotomètre $\mathrm{DR} / 5000$.

Résultants :-

Le tableau 1 montre les résultats d'analyse physicochimiques de l'eau brute du Fleuve Sénégal enregistrées entre Janvier et Septembre2016.

Tableau 1:-Résultats physicochimiques de l'eau brute du Fleuve Sénégal enregistrées du Janvier au Septembre 2016

\begin{tabular}{|c|c|c|c|c|c|c|c|c|c|c|c|c|c|}
\hline & $\begin{array}{l}\mathrm{T} \\
{ }^{\circ} \mathrm{C}\end{array}$ & $\mathrm{pH}$ & $\begin{array}{l}\text { Cond. } \\
\mu \mathrm{S} / \mathrm{cm}\end{array}$ & $\begin{array}{l}\text { Turb. } \\
\text { NTU }\end{array}$ & $\begin{array}{l}\mathrm{MO} \\
\mathrm{mg} / \text {. } \\
\mathrm{L}\end{array}$ & $\begin{array}{l}\mathrm{NO}^{-} 3 \\
\mathrm{mg} / \\
\mathrm{L}\end{array}$ & $\begin{array}{l}\mathrm{NO}^{-} 2 \\
\mathrm{mg} / \\
\mathrm{L}\end{array}$ & $\begin{array}{l}\text { TAC } \\
{ }^{\circ} \mathrm{F}\end{array}$ & $\begin{array}{l}\text { TH } \\
{ }^{\circ} \mathrm{F}\end{array}$ & $\begin{array}{l}\mathrm{MES} \\
\mathrm{mg} / \mathrm{L}\end{array}$ & $\begin{array}{l}\mathrm{Cl} \\
\mathrm{Mg} / . \\
\mathrm{L}\end{array}$ & $\begin{array}{l}\mathrm{O}_{2} \\
\text { mg./ } \\
\mathrm{L} .\end{array}$ \\
\hline \multirow{5}{*}{ 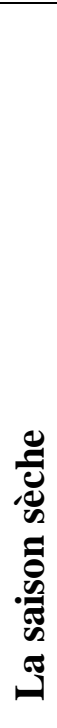 } & $\underset{\Xi}{\Xi}$ & 18.9 & $\begin{array}{l}7 . \\
9\end{array}$ & $\begin{array}{c}57 . \\
1\end{array}$ & $\begin{array}{c}43 . \\
2\end{array}$ & 0.9 & $\begin{array}{c}0.7 \\
0\end{array}$ & $\begin{array}{c}0.01 \\
6\end{array}$ & $\begin{array}{l}4 . \\
4\end{array}$ & 2 & $\begin{array}{l}3 \\
2\end{array}$ & 14.4 & $\begin{array}{c}6.7 \\
8\end{array}$ \\
\hline & $\dot{\bar{d}}$ & 19 & $\begin{array}{l}7 . \\
8\end{array}$ & 57 & $\begin{array}{c}42 . \\
8\end{array}$ & 0.8 & $\begin{array}{c}0.6 \\
0\end{array}$ & $\begin{array}{c}0.01 \\
7\end{array}$ & $\begin{array}{l}4 . \\
4\end{array}$ & 2 & $\begin{array}{l}3 \\
0\end{array}$ & 14.2 & $\begin{array}{c}6.8 \\
0\end{array}$ \\
\hline & $\sum_{\Sigma}^{\bar{z}}$ & $\begin{array}{c}25 . \\
8\end{array}$ & $\begin{array}{l}7 . \\
5\end{array}$ & $\begin{array}{c}54 . \\
5\end{array}$ & $\begin{array}{c}25 . \\
8\end{array}$ & 2.2 & $\begin{array}{c}0.7 \\
6\end{array}$ & $\begin{array}{c}0.01 \\
2\end{array}$ & $\begin{array}{l}3 . \\
6\end{array}$ & 1. & $\begin{array}{l}2 \\
3\end{array}$ & $\begin{array}{c}11.5 \\
2\end{array}$ & $\begin{array}{c}6.7 \\
0\end{array}$ \\
\hline & $\frac{3}{2}$ & 23 & $\begin{array}{l}7 . \\
6\end{array}$ & 55 & 26 & 2.4 & $\begin{array}{c}0.8 \\
0\end{array}$ & $\begin{array}{c}0.01 \\
4\end{array}$ & $\begin{array}{l}3 . \\
8\end{array}$ & $\begin{array}{l}1 . \\
7\end{array}$ & $\begin{array}{l}2 \\
4\end{array}$ & $\begin{array}{c}11.6 \\
0\end{array}$ & $\begin{array}{c}6.8 \\
1\end{array}$ \\
\hline & $\sum^{\pi}$ & $\begin{array}{c}20 . \\
8\end{array}$ & $\begin{array}{l}7 . \\
2\end{array}$ & $\begin{array}{c}52 . \\
2\end{array}$ & $\begin{array}{c}21 . \\
3\end{array}$ & 1.9 & $\begin{array}{c}0.8 \\
0\end{array}$ & $\begin{array}{c}0.01 \\
8\end{array}$ & $\begin{array}{l}2 . \\
4\end{array}$ & $\begin{array}{l}2 . \\
4\end{array}$ & $\begin{array}{l}2 \\
8\end{array}$ & $\begin{array}{c}17.2 \\
3\end{array}$ & $\begin{array}{c}6.4 \\
6\end{array}$ \\
\hline \multirow{4}{*}{ 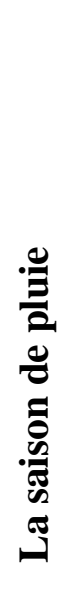 } & $\Xi$ & 29 & $\begin{array}{l}7 . \\
2\end{array}$ & 54 & 27 & 1.2 & 3.1 & $\begin{array}{c}0.01 \\
0\end{array}$ & $\begin{array}{l}3 . \\
5\end{array}$ & 1. & 8 & 2.3 & $\begin{array}{c}5.7 \\
8\end{array}$ \\
\hline & $\stackrel{\Xi}{\Xi}$ & 29.7 & $\begin{array}{l}7 . \\
3\end{array}$ & 51 & $\begin{array}{c}26 . \\
9\end{array}$ & 1.2 & 3.1 & $\begin{array}{c}0.01 \\
2\end{array}$ & $\begin{array}{l}3 . \\
6\end{array}$ & 1. & 9 & 2.4 & $\begin{array}{c}5.8 \\
8\end{array}$ \\
\hline & 总 & 28.9 & $\begin{array}{l}7 . \\
2\end{array}$ & $\begin{array}{c}67 . \\
1\end{array}$ & $\begin{array}{c}33 \\
0\end{array}$ & 3.4 & 3.7 & $\begin{array}{c}0.01 \\
9\end{array}$ & $\begin{array}{l}3 . \\
6\end{array}$ & $\begin{array}{l}1 . \\
6\end{array}$ & $\begin{array}{c}27 \\
0\end{array}$ & 2.4 & $\begin{array}{c}3.9 \\
5\end{array}$ \\
\hline & 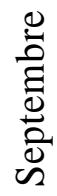 & 27.8 & $\begin{array}{l}7 . \\
1\end{array}$ & $\begin{array}{c}47 . \\
4\end{array}$ & $\begin{array}{c}15 \\
8\end{array}$ & 2.5 & 5.5 & $\begin{array}{c}0.01 \\
0\end{array}$ & $\begin{array}{l}3 . \\
2\end{array}$ & $\begin{array}{l}5 . \\
6\end{array}$ & $\begin{array}{c}14 \\
2\end{array}$ & 2 & $\begin{array}{c}3.7 \\
7\end{array}$ \\
\hline
\end{tabular}




\section{Interprétation des résultants:-}

Température et $\mathbf{p H}$ :-

La température de l'eau régit presque la totalité des réactions physiques, chimiques et biologiques [1]. Elle joue un rôle important dans la solubilité des sels et surtout des gaz et conditionne les équilibres de dissociation. En effet, toute variation brusque de ce paramètre entraine une perturbation dans l'équilibre de l'écosystème aquatique. La variation de la température entre les saisons de l'année montre une valeur élevée dans la saison de pluie alors qu'elle atteint $29.7^{\circ} \mathrm{C}$ au mois de juillet (figure2). Le pH influence fortement le mécanisme des réactions chimiques et biologiques dans les eaux. Dans les eaux naturelles, les valeurs du pH se situent entre 6 et 8.5 dans les eaux naturelles. Il diminue en présence des teneurs élevées en matière organique et augmente en période d'étiage, lorsque l'évaporation est importante [1]. Les valeurs du pH (Figure3) ne montrent pas de différences durant les mois de Mai, Juillet, Aout et Septembre. La valeur maximale de pH qui est autour de 7.9 est obtenue pour le mois de Janvier (figure 3). Les valeurs de $\mathrm{pH}$ suivis durant 9 mois ne montrent pas d'effets négatifs sur la production d'eau potable.

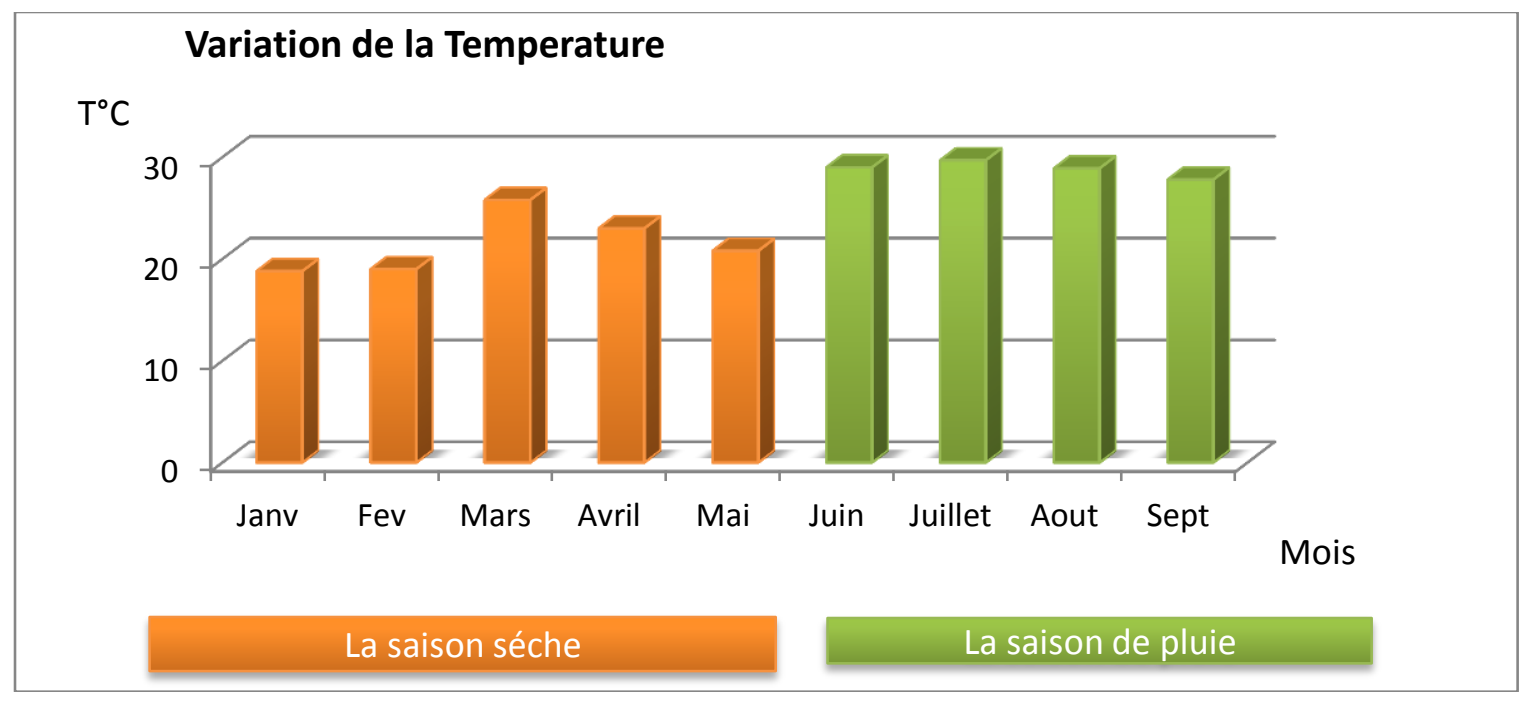

Figure 2:- Variation de la température

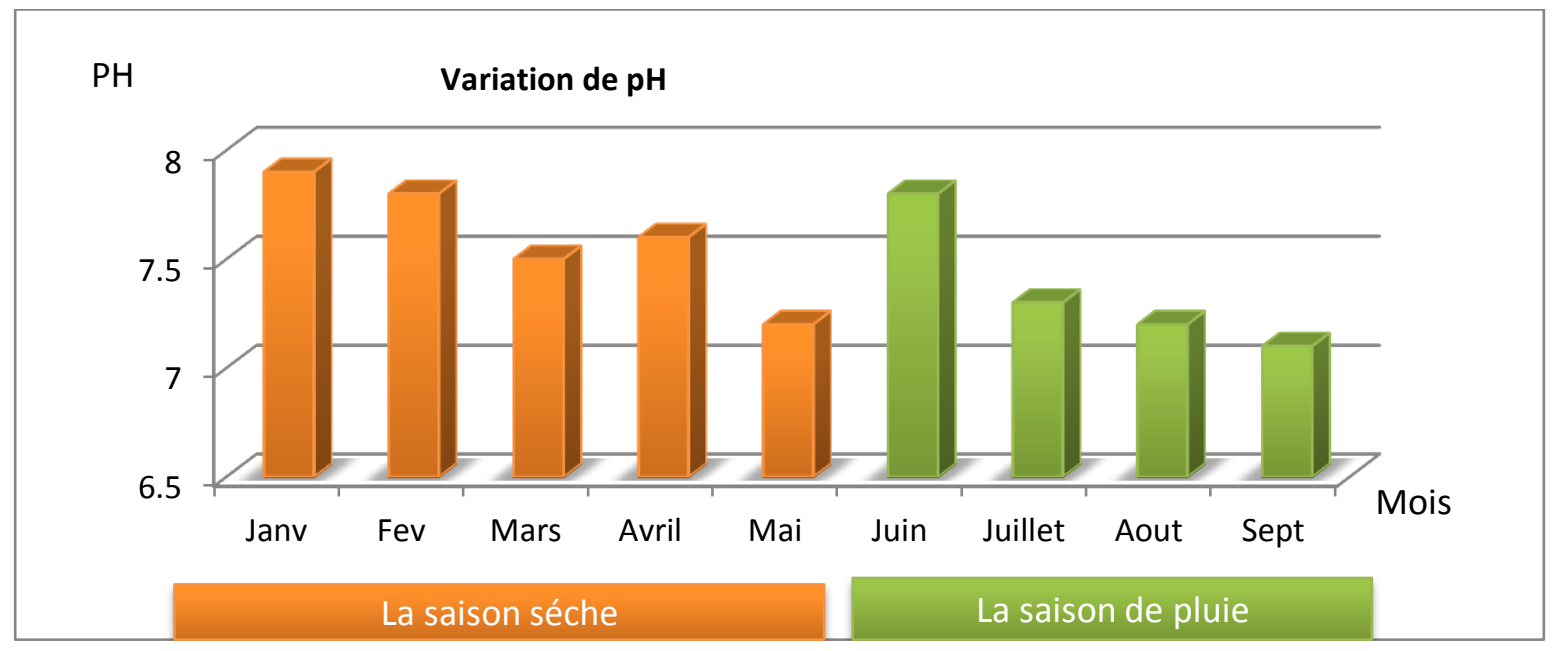

Figure 3:- Variation du $\mathrm{pH}$

La turbidité et la conductivité électrique :-

La turbidité d'une eau est due à la présence des matières en suspension finement divisées : argile, limons, grains de silice, matières organiques, etc. L'appréciation de l'abondance de ces matières mesure son degré de turbidité. La variation de la turbidité entre les saisons de l'année (figure 4) ne montre pas une variation importante durant les 
mois de Janvier, Février, Mars, Avril, Mai, Juin et Juillet où elle varie entre 21.3 et 43.2 NTU tandis que des valeurs importantes ont été mesurés durant les mois d'Aout et Septembre avec une valeur maximale de 330NTU (figure 4) au mois d'Aout ce qui peut être du au drainage des eaux pluviales vers le fleuve. Selon les données bibliographiques on trouve des valeurs très élevées au niveau du captage de la Station de Traitement de Béni Nadji au bord du fleuve Sénégal oscillant entre 3.15 et 980 NTU [8]. Ces teneurs de turbidité enregistrées dépassent largement la valeur limite acceptable pour les eaux destinées à la consommation humaine qui est de 5 NTU OMS[9].

La conductivité électrique (EC) est une expression numérique de la capacité d'une solution à conduire le courant électrique. La plupart des sels minéraux en solution sont de bons conducteurs. Par contre, les composés organiques sont des mauvaises conductrices. En effet, plus la conductivité est élevée plus l'eau est fortement minéralisé. La conductivité d'une eau naturelle est comprise entre 50 et $1500 \mu \mathrm{S} / \mathrm{cm}$.

La variation de la conductivité électrique entre les saisons de l'année (figure 4) ne montre pas une variation importante (47.4 et $67.1 \mu \mathrm{S} / \mathrm{cm})$

En comparant les valeurs de la conductivité détectées au niveau des eaux étudiées et celles mesurés d'Oued Sebou (Maroc) qui peut atteindre $2700 \mu \mathrm{s} / \mathrm{cm}$ [2] on trouve que les eaux du fleuve Sénégal est de bonne qualité par rapport à la qualité des eaux de surfaces d'Oued Sebou recevant des rejets industriels sans traitement.(la conductivité électrique dépend aussi de la nature lithologique du bassin versant).

La conductivité électrique devrait diminuer pendant la saison des pluies (effet de dilution). Mais en 2016 la conductivité électrique n'a pas varié considérablement à cause de faibles précipitations pendant la saison de pluies.

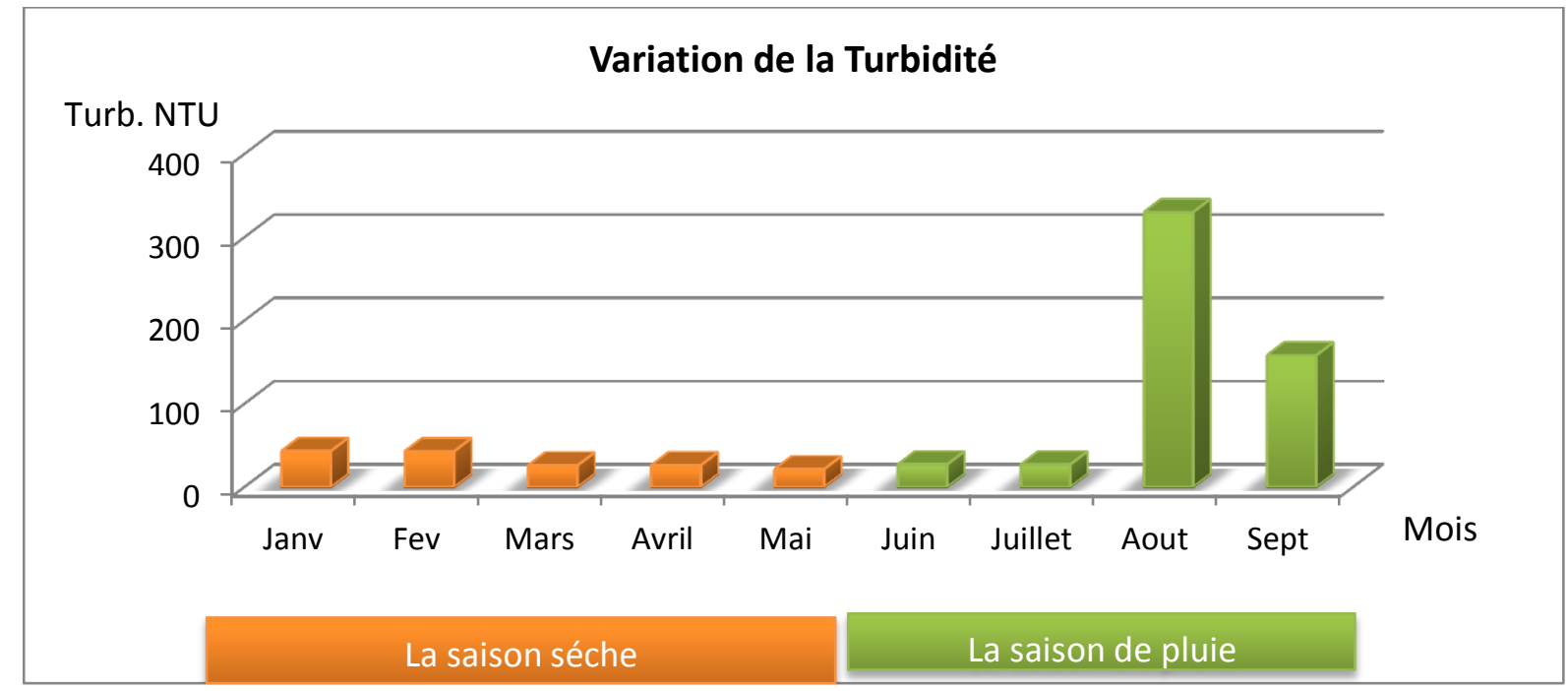

Figure 4:- Variation de la turbidité 


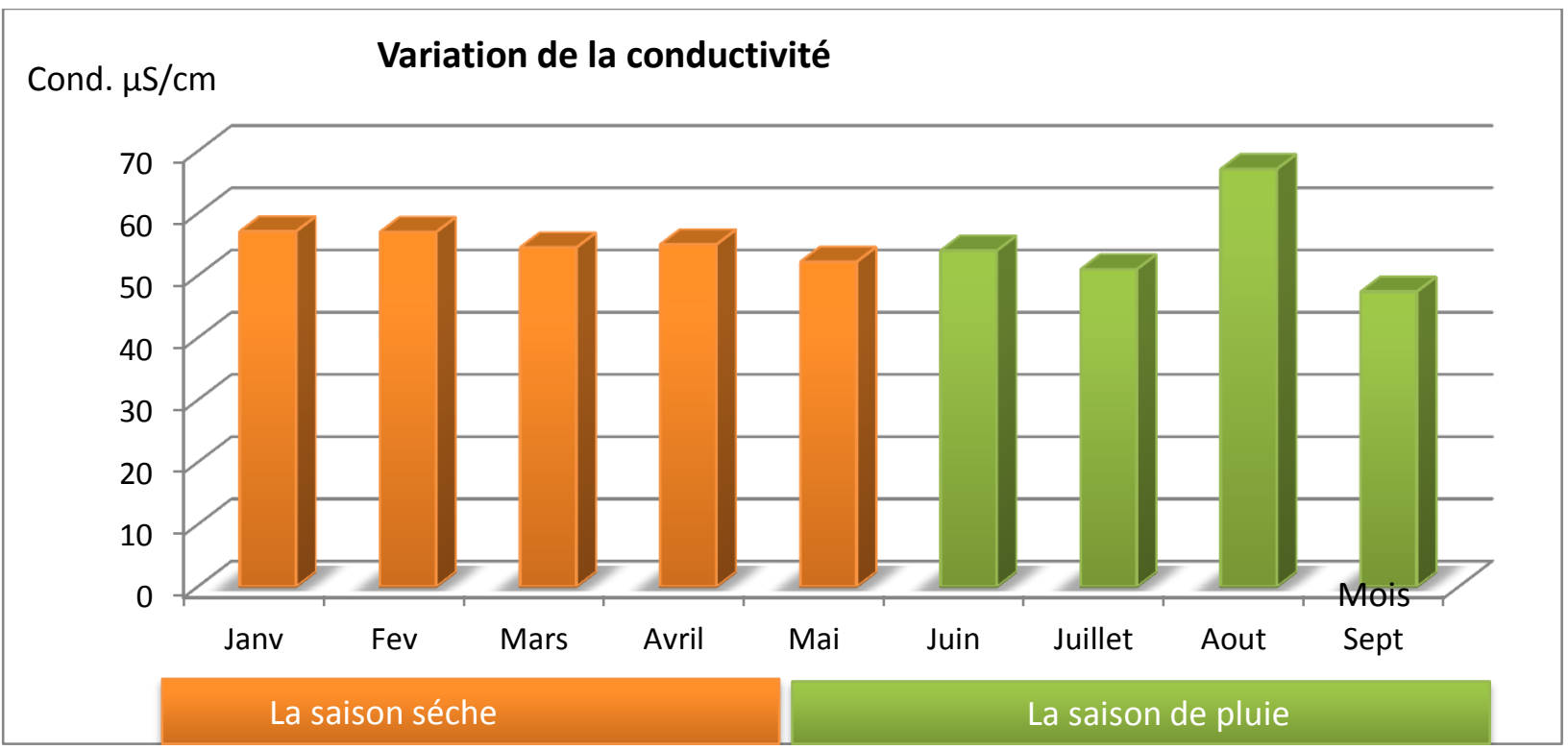

Figure 5:- Variation de la conductivité

\section{Nitrates et Nitrites:-}

Dans certains cas, les nitrates constituent la forme azotée la plus dominante dans les cours d'eau et matière organique par oxydation bactérienne des nitrites et constituent ainsi l'ultime produit de la nitrification. En milieu naturel, sa concentration dépasse rarement $0.45 \mathrm{mg} . \mathrm{L}^{-1}$ [10]. Des valeurs supérieures indiquent des rejets d'eaux usées dans les milieux aquatiques superficielles et souterraines, et surtout une utilisation excessive de fertilisants utilisés en agriculture [10].

La variation annuelle des nitrates (Figure 6) montre des valeurs minimales dans les mois de Janvier, Février, Mars, Avril et Mai avec des concentrations inférieures à $0.8 \mathrm{mg} / \mathrm{L}$ et des valeurs plus importantes dans les mois de Juillet, Aout et Septembre où il passe de $3.1 \mathrm{mg} / \mathrm{L}$ au mois de Juillet à $5.5 \mathrm{mg} / \mathrm{L}$ au mois de Septembre (figure 6). Les concentrations en nitrates sont trop faible dans les eaux de surface et ne pose aucun problème pour la potabilisation (figure 6).

En effet, le nitrate est la forme la plus stable de l'azote minéral dans le milieu naturel en conditions aérobies. Cet élément présente des risques pour la santé. Des études ont montré son caractère cancérigène [11]. Alors que d'autres épidémiologistes réfutent ce lien. Par ailleurs, l'azote qui est un élément indispensable à la croissance des végétaux, induit une perturbation des écosystèmes aquatiques [12].

L'azote est présent sous différentes formes dans le sol :

* L'azote minéral : nitrate, nitrite et ammonium sont en phase aqueuse ou adsorbée. La forme minérale représente de l'ordre de 2 à $4 \%$ de l'azote total du sol, c'est une forme soluble hautement lessivable;

* L'azote organique est intégré dans les organismes (racines, microflore et microfaune) et dans la matière organique du sol. Il s'agit d'une forme de l'azote qui migre difficilement dans le milieu. Sa quantité dépend du type de sol et de la biomasse.

La dynamique de la minéralisation du stock organique (qui représente de l'ordre de 96 à $98 \%$ de l'azote du sol) est par ailleurs sujette aux variations climatiques puisqu'elle est fonction des températures et de la teneur en eau. Conformément à ces phénomènes, les flux et les concentrations les plus importantes en nitrates s'observent généralement en automne lorsqu'il fait doux, que les pluies sont efficaces et que les besoins des végétaux chutent. Plus la lame d'eau écoulée est importante, plus les flux sont élevés : les années humides sont donc des années de forte pollution nitratée. Le drainage des sols accroît le potentiel de lessivage de ceux-ci et conduit à une augmentation des flux de nitrates dans les eaux [13]. 
Les régions les plus touchées par la pollution azotée sont celles d'élevage intensif à forte charge en azote organique due aux effluents animaux, celles de grandes cultures fortement fertilisées et celles où les ressources en eau sont superficielles ou de faible profondeur (zones de socle aux sous-sols peu perméable, nappes aquifères alluviales, nappes aquifères karstiques) [14].

Les nitrites peuvent être rencontrés dans les eaux, mais généralement à des concentrations faibles, une eau qui renferme des nitrites peut être considérée comme suspect. Il semble qu'une teneur d'azote nitreux supérieur à 0.10 $\mathrm{mg} . \mathrm{L}^{-1}$ (figure7) puisse faire soupçonner un apport d'eaux riches en matières organiques en voie d'oxydation. Cette concentration ne devrait pas être dépassée dans une eau d'origine profonde [10]. es variations de nitrite entre les saisons de l'année (Figure7) ne montrent pas des différences significatives, globalement il varie entre 0.010 et $0.019 \mathrm{mgL}^{-1}$.

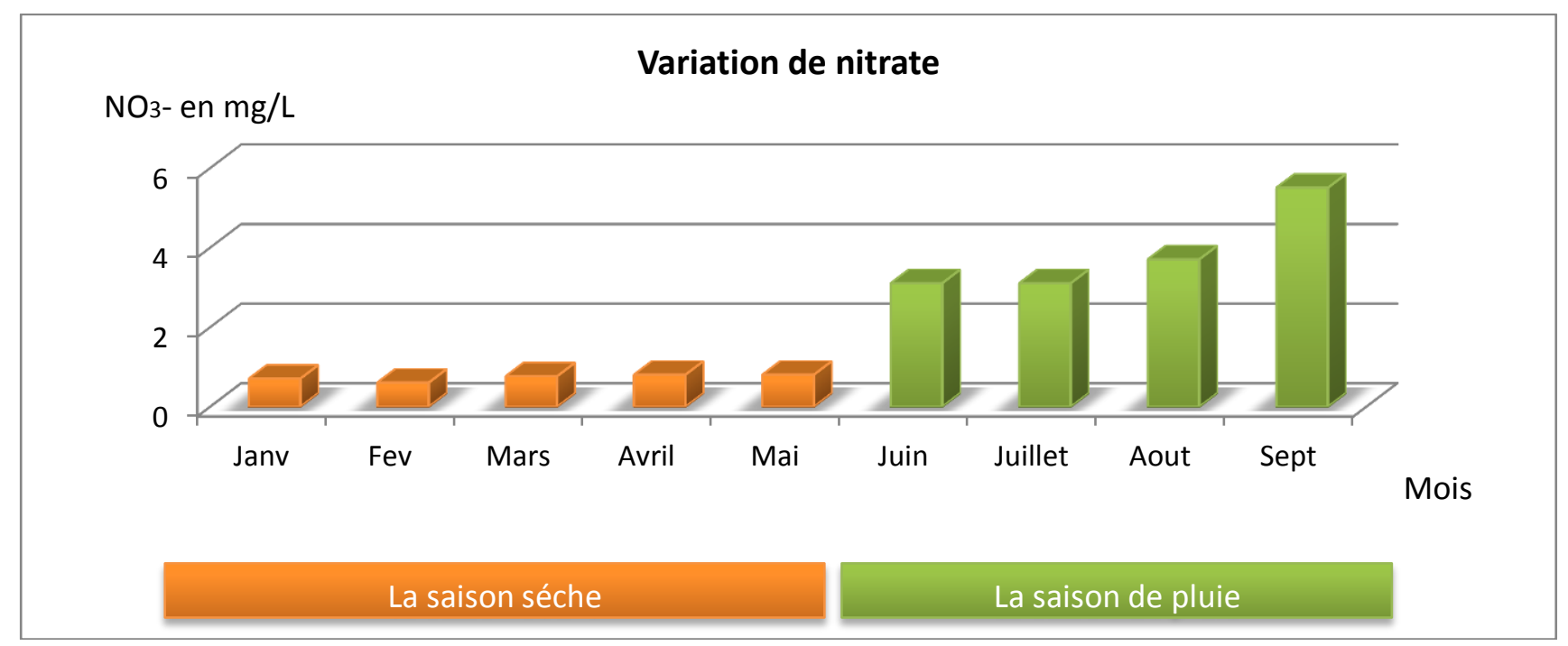

Figure 6:- Variation du nitrate (NO3)

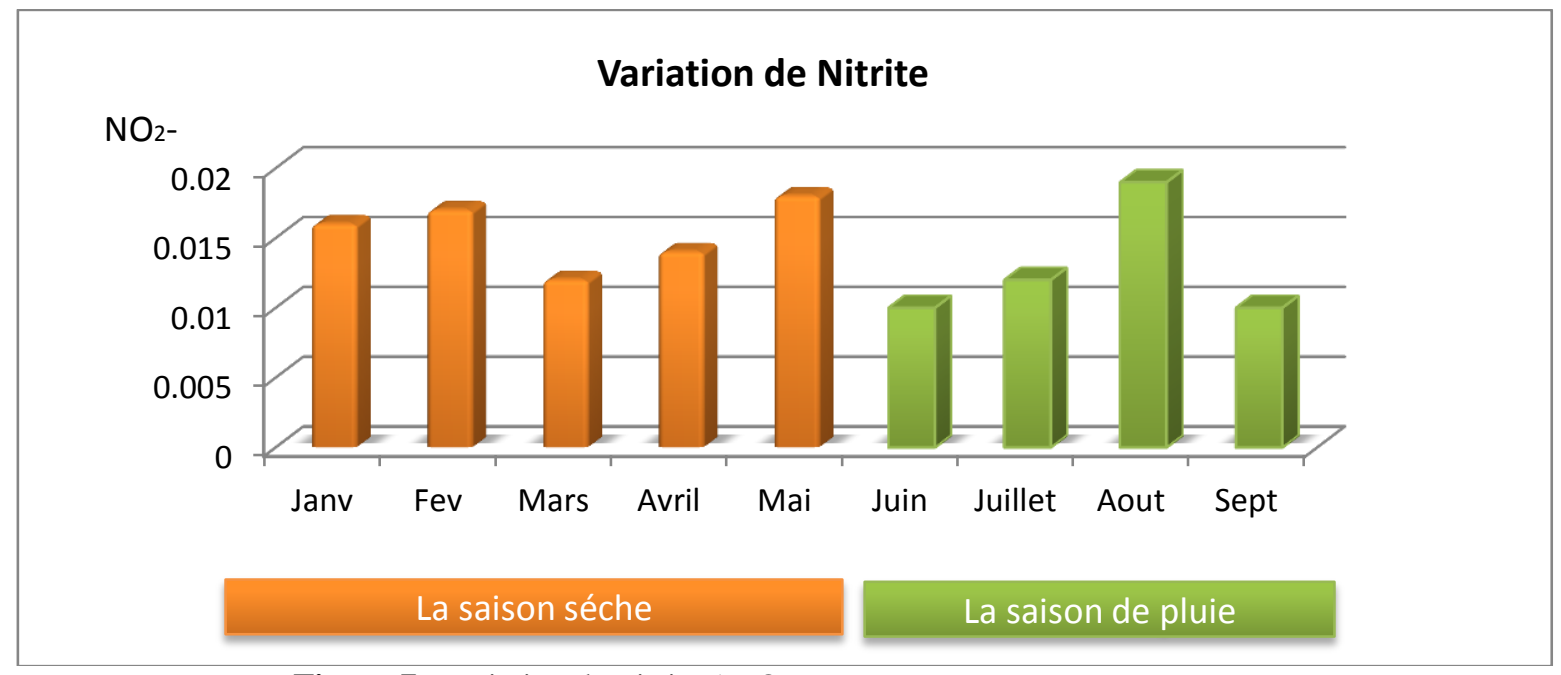

Figure 7: Variation du nitrite ( $\left.\mathrm{NO}_{2}\right)$

Les Matières en suspension et la matière organique :-

Les matières en suspension comprennent toutes les matières minérales ou organiques qui sont insolubles dans l'eau. Elles incluent les argiles, les sables, les limons, les matières organiques et minérales de faible dimension, le plancton et autres micro-organismes de l'eau. La quantité de matières en suspension varie notamment selon les saisons et le régime d'écoulement des eaux. Ces matières affectent la transparence de l'eau et diminuent la 
pénétration de la lumière et, par suite, la photosynthèse. Elles peuvent également gêner la respiration des poissons. Par ailleurs, les matières en suspension peuvent accumuler des quantités élevées de matières toxiques telles que les métaux, pesticides, huiles minérales, hydrocarbures aromatiques polycyclique, etc.

La variation annuelles de MES (figure 8) montre une variation considérable durant les mois de Janvier, février, Mars, Avril, Mai, et Juillet où elle varie entre $9 \mathrm{mg} / \mathrm{L}$ au mois de Juillet et $32 \mathrm{mg} / \mathrm{L}$.Ces variations importantes ont été mesuré durant les mois de d'Aout et Septembre avec une valeur maximale de $275 \mathrm{mg} / 1$ au mois d'Aout (figure 8).

la matière organique : Présente dans l'eau peut avoir une origine naturelle (activité biologique des milieux hydriques : décompositions des végétaux et des microorganismes, etc) ou anthropique (pollution domestique, industrielle ou agricole). Les eaux de surface présentent en général une oxydabilité comprise entre 3 et $12 \mathrm{mg} / \mathrm{L}$. La variation de l'oxydabilité entre les saisons de l'année (Figure 9) montre des valeurs minimales dans les mois de Janvier, février, Mars, Avril, Mai, Juin, Juillet Septembre où elle varie entre 0.8 et $2.5 \mathrm{mg} / \mathrm{Let}$ une valeur maximale de $3.4 \mathrm{mg} / \mathrm{lau}$ mois d'Aout (figure 9).

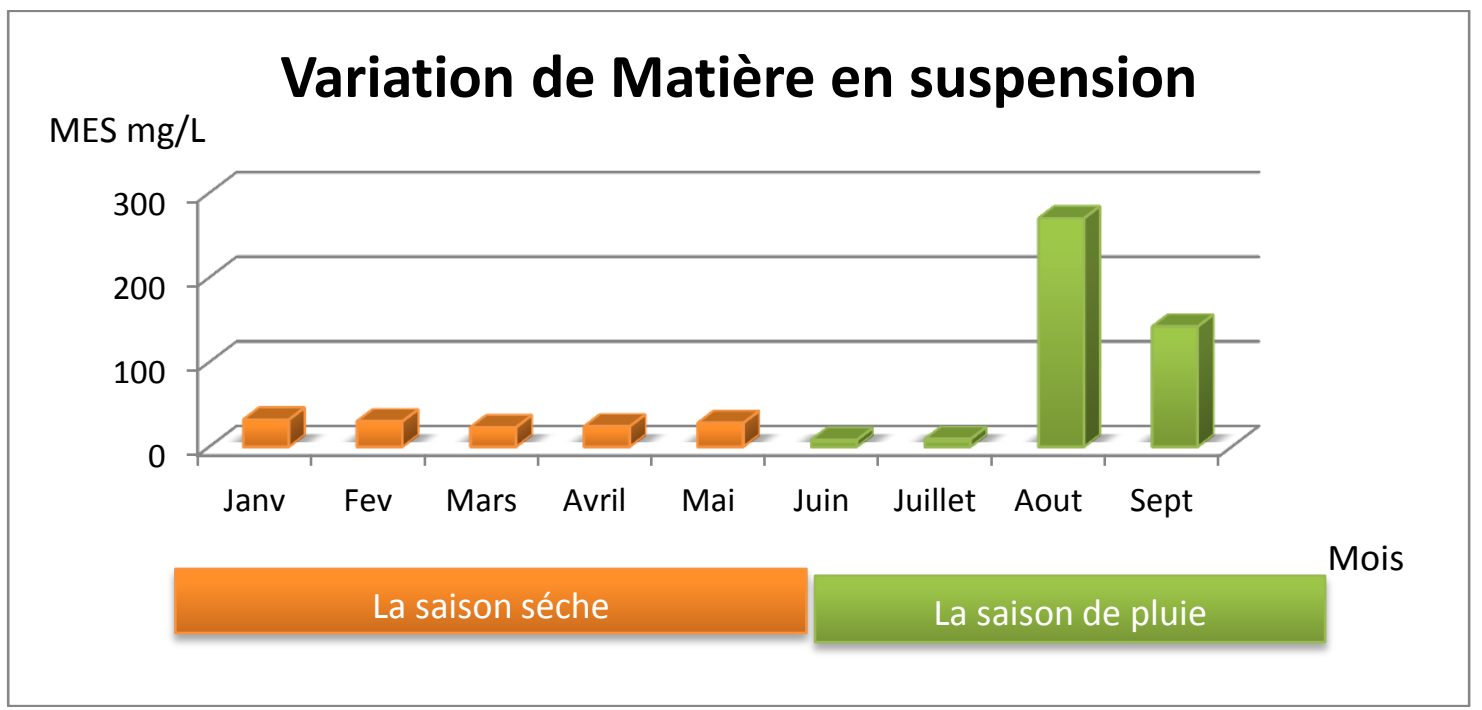

Figure 8:- Variation de la MES

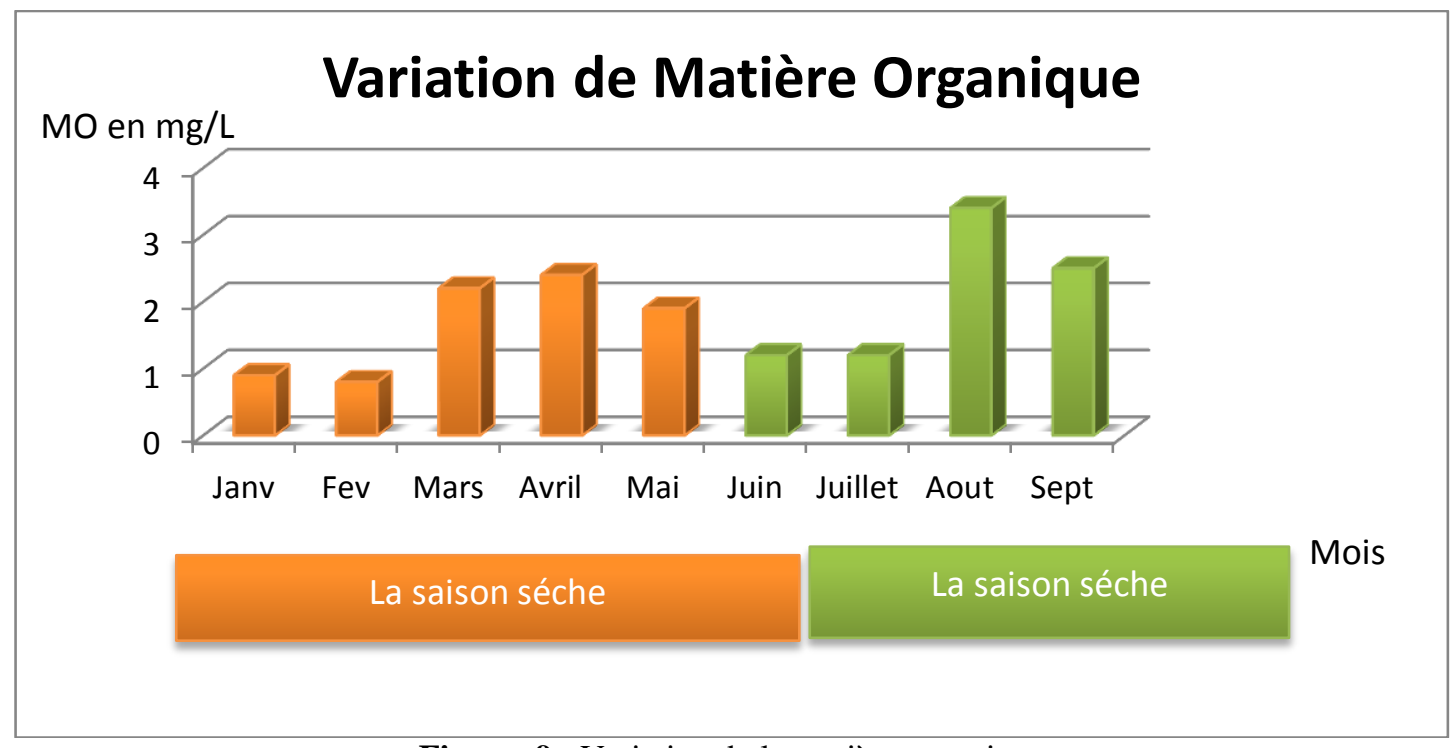

Figure 9: Variation de la matière organique 


\section{L'oxygène dissous :-}

L'oxygène dissous est l'un des plus importants paramètres de qualité des eaux pour la vie aquatique. Il provient essentiellement de l'atmosphère et de l'activité photosynthétique des algues et des plantes aquatiques. La concentration en oxygène dissous varie de manière journalière et saisonnière car elle dépend de nombreux facteurs tels que la pression partielle en oxygène de l'atmosphère, la température de l'eau, la salinité, la pénétration de la lumière, l'agitation de l'eau et la disponibilité en nutriments. Cette concentration en oxygène dissous est également fonction de la vitesse d'appauvrissement du milieu en oxygène par l'activité des organismes aquatiques, les processus d'oxydation et de décomposition de la matière organique présente dans l'eau [15].

La variation d'O2 entre les saisons de l'année (Figure 10) montre que les eaux du fleuve Sénégal sont plus oxygénées durant les mois de Janvier, février, Mars, et Mai, où elle varie entre 6.46 et $6.81 \mathrm{mg} / \mathrm{L}$. En effet, les eaux du fleuve Sénégal sont moins oxygénées durant les mois Juin, Juillet d'Aout et Septembre où elle varie entre 3.77 et $5.78 \mathrm{mg} / \mathrm{L}$ avec une valeur minimale de $3.77 \mathrm{mg} . \mathrm{L}^{-1}$ au mois de Septembre. Les eaux de surfaces étudiées appartiennent à la classe de qualité bonne à mauvaise.

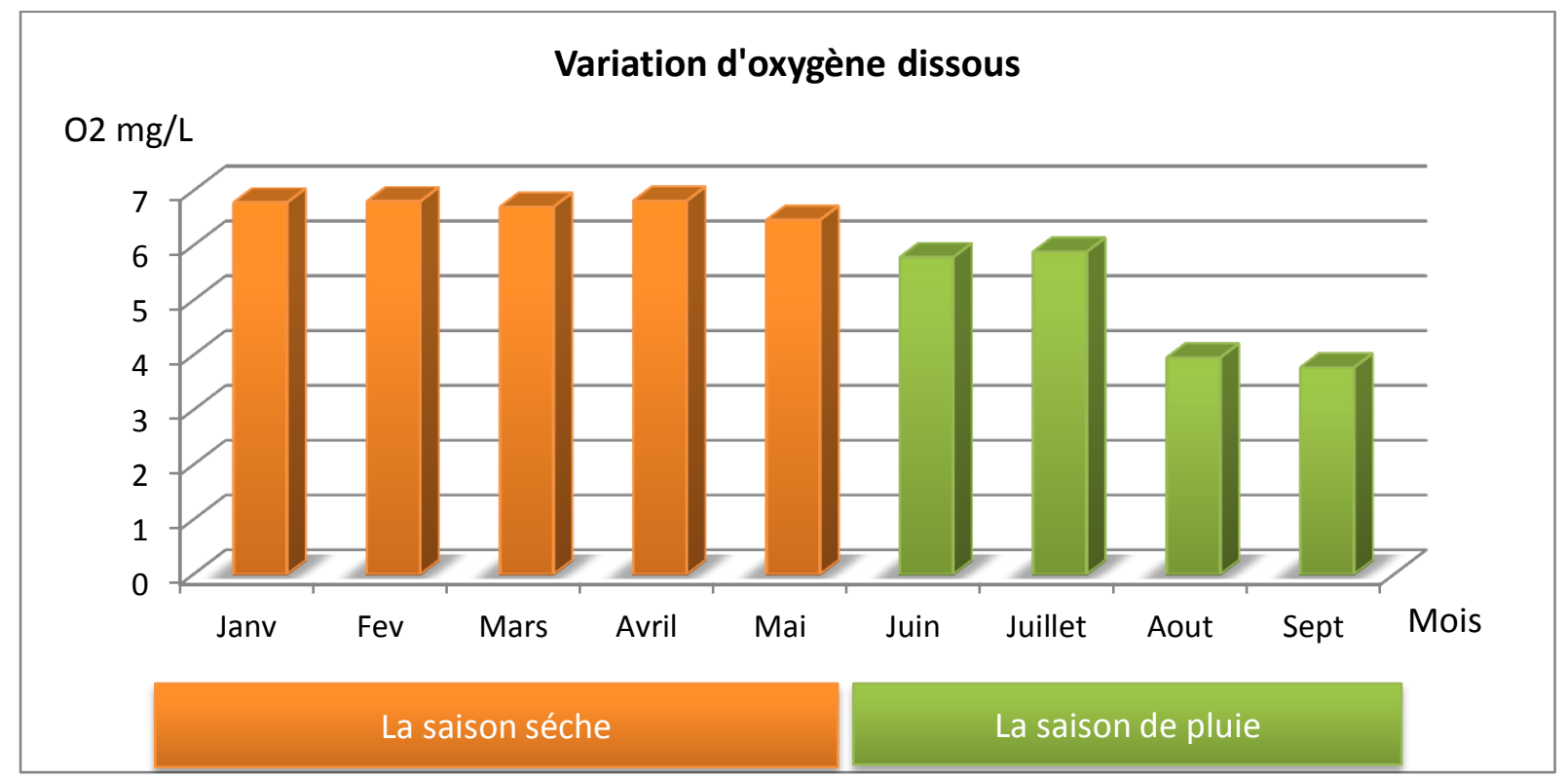

Figure 10:- Variation d'Oxygène dissous entre les saisons de l'année.

\section{Le TAC et la Dureté totale :-}

Le TAC (titre alcalimétrique complet) est la grandeur utilisée pour mesurer le taux d'hydroxydes, de carbonates et de bicarbonates d'une eau, son unité est le degré français $\left({ }^{\circ} \mathrm{f}\right)$. Il correspond à la neutralisation de la totalité des espèces carboniques. C'est une mesure globale évaluant en bloc les carbonates et bicarbonate alcalins et alcalinoterreux éventuellement, borates, silicates phosphates alcalins.

La variation de TAC entre les saisons de l'année (Figure12) ne montre pas une variation durant les mois de Mars, Juillet, Aout et Septembre où elle varie entre 3.6 et $3.2 \mathrm{~F}^{\circ}$ tandis que des variations importantes ont été mesurées durant les mois de Mai avec une valeur minimale de $2.4 \mathrm{~F}^{\circ}$ et Janvier avec une valeur maximale de $4.4^{\circ} \mathrm{F}$. La dureté totale représente la teneur de l'eau en sels de métaux alcalino-terreux (sels de calcium, magnésium, strontium et baryum). Etant donné que le strontium et le baryum sont souvent présents dans l'eau sous forme de traces, la dureté totale se réduit à sa concentration en ions calcium et magnésium, exprimés en millimoles ou en milligrammes par litre (mmol/l ou mg. $\left.\mathrm{L}^{-1}\right)$ ou en degré français $\left({ }^{\circ} \mathrm{F}\right)[10]$.

Le titre hydrotimétrique $(\mathrm{TH})$ ou dureté [totale] de l'eau $(\mathrm{Ca}+\mathrm{Mg})$, est l'indicateur de la minéralisation de l'eau. Elle est surtout due aux ions calcium et magnésium 
La variation de TH entre les saisons de l'année (Figure 11) ne montre pas une variation durant les mois de Janvier, Mars, Juillet et Aout où elle varie entre 1.6 et $2.4 \mathrm{~F}^{\circ}$ tandis que la variation importante a été mesurée durant le mois de Septembre avec une valeur maximale de $5.6 \mathrm{~F}^{\circ}$ (figure 11 ).

Le tableau 2, montre les différents types d'eau en fonction de la valeur de la dureté (TH).

Tableau 2:- Plage de valeurs du titre hydrotimétrique.

\begin{tabular}{|l|l|l|l|l|l|}
\hline TH $\left({ }^{\circ} \mathrm{f}\right)$ & 0 à 7 & 7 à 15 & 15 à 25 & 25 à 42 & supérieur à 42 \\
\hline Eau & très douce & douce & moyennement dure & Dure & très dure \\
\hline
\end{tabular}

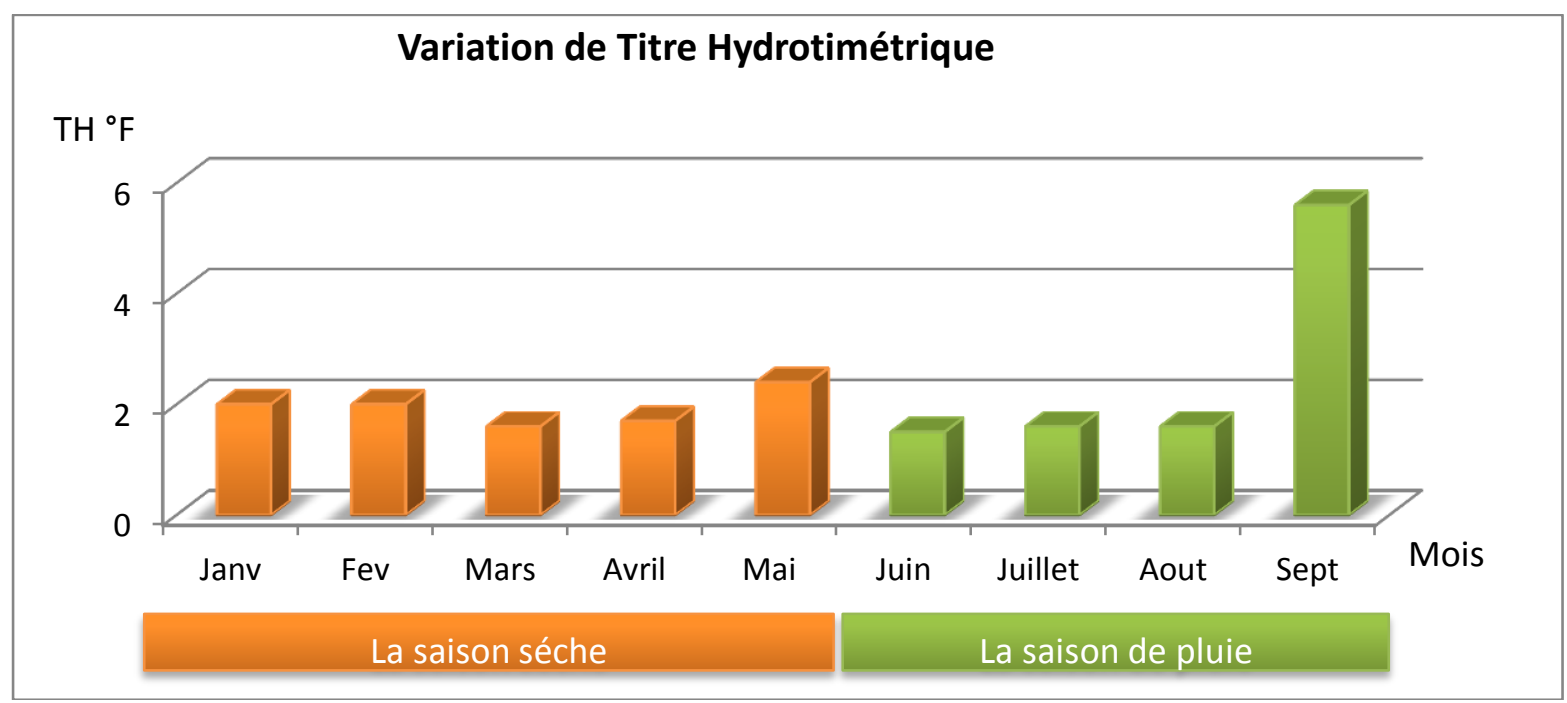

Figure 11:- Variation de TH

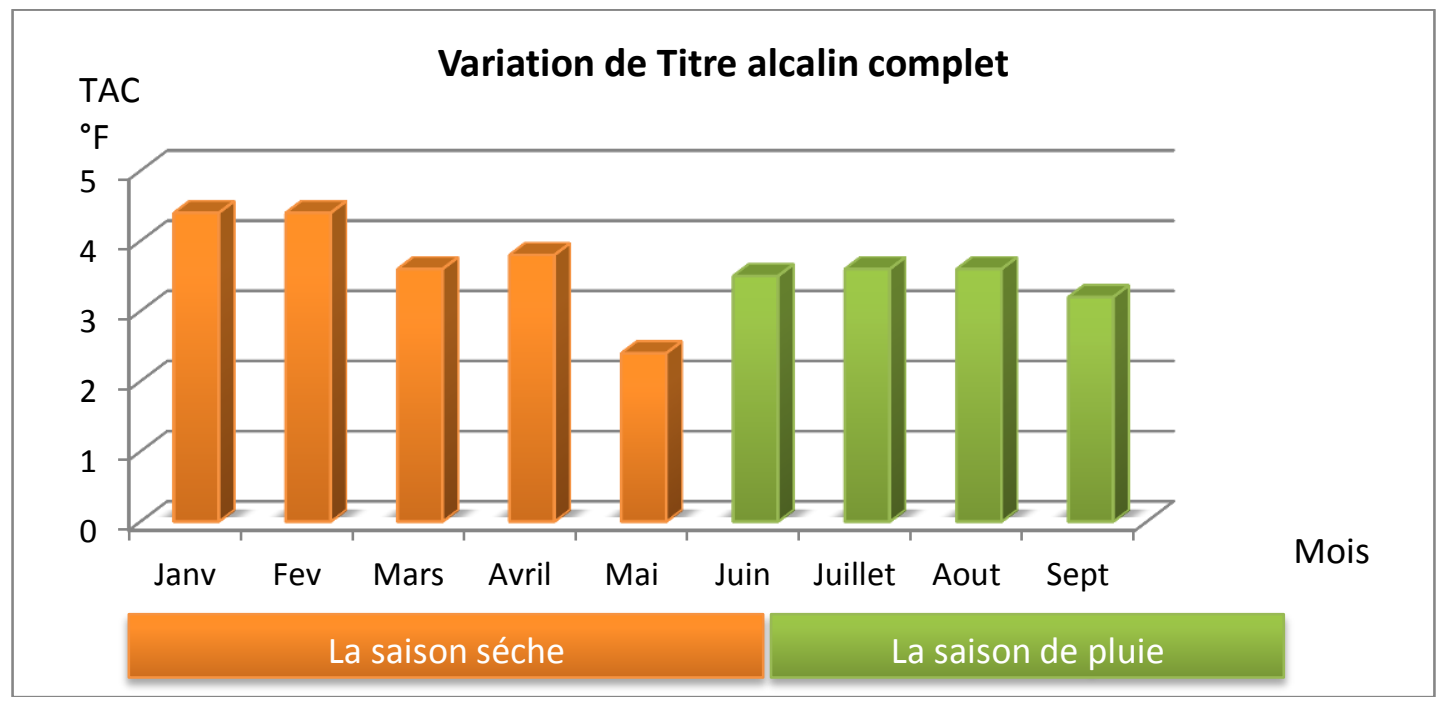

Figure 12 : Variation du titre alcalin Complet

\section{Les chlorures :-}

Les teneurs en chlorures des eaux sont extrêmement variées et liées principalement à la nature des terrains traversés. Habituellement, la teneur en ion chlorure des eaux naturelles est inférieure à $50 \mathrm{mg} / \mathrm{l}$, mais elle peut subir des variations provoquées dans les zones arides par lessivage et dans les zones industrielles par des pollutions des eaux usées. Le principal inconvénient des chlorures est la saveur désagréable qu'ils communiquent à l'eau. La réglementation marocaine suggère que la teneur en chlore des eaux ne dépasse pas $300 \mathrm{mg} . \mathrm{L}^{-1}$. La variation des chlorures entre les saisons de l'année (Figure 13) des eaux du fleuve Sénégal ne montre pas une variation 
significative durant les mois de Juin, Juillet, Aout et Septembre où elle varie entre 2 et $2.4 \mathrm{mg} . \mathrm{L}^{-1}$ tandis que des valeurs importantes ont été mesurées durant le mois de Janvier, février, Mars, Avril et Mai avec des valeurs respectivement $14.4,14.2,11.52,11.60$, et $17.23 \mathrm{mg} . \mathrm{L}^{-1}$ (figure13).

D'après l'étude réalisée par Troussellier and al [16]. Les caractéristiques des eaux saumâtres le long du fleuve varient en fonction de point de prélèvement. Dans certains points la salinité atteint la valeur de la salinité des océans $\left(35\right.$ g. $\left.\mathrm{L}^{-1}\right)$. En outre, durant l'étude réalisée sur le fleuve sénégalais par Gisèle and al [17], il a été rapporté que la concentration en sel et la température varient au cours du temps et d'un point de prélèvements à un autre.

$\mathrm{Cl} \mathrm{mg} / \mathrm{L}$

\section{Variation de Chlorure}

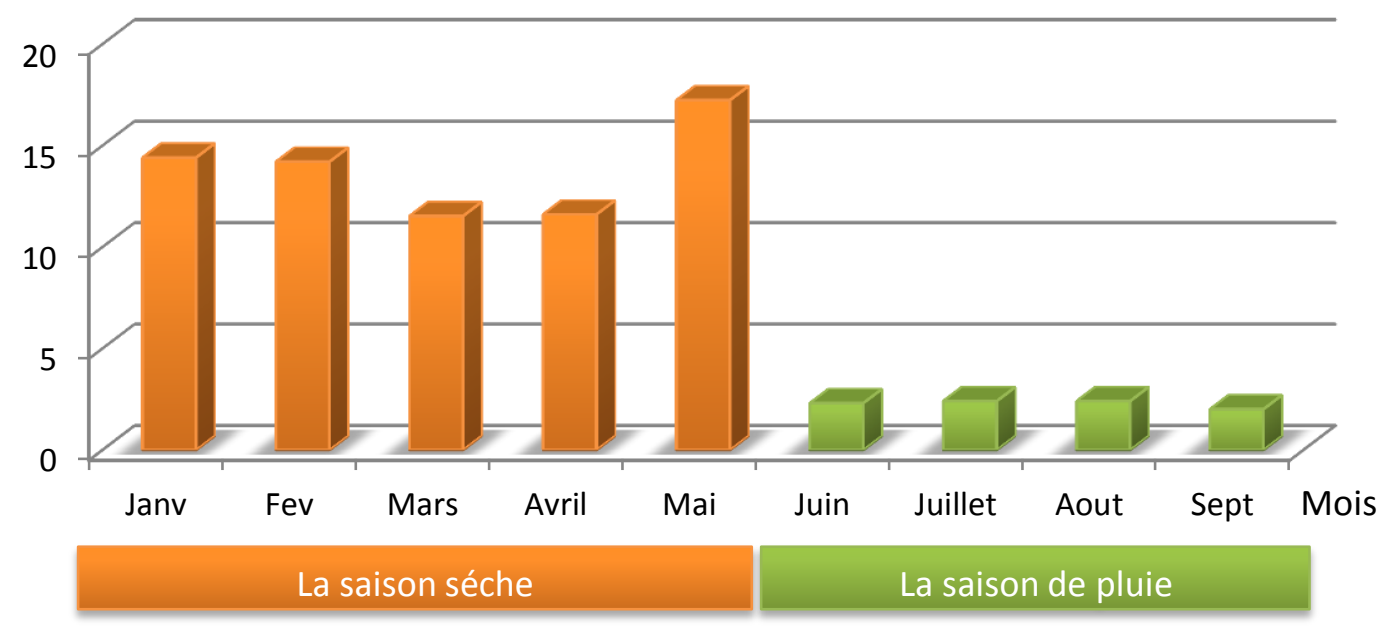

Figure 13:- Variation de Chlorure

\section{Analyse en Composantes Principales d'eau Aftout Essahili :-}

Dans le but d'établir une relation entre les différents paramètres physicochimiques et pour mieux évaluer l'effet des changements saisonniers sur la qualité des eaux du site de la prise d'eau d'AftoutEssahili, un traitement statistique ACP (analyse en composantes principales) sur le logiciel statistique IBM SPSS Statistics 20, a été appliqué à l'ensemble des paramètres en saison pluviale et en saison sèche. Cette méthode est largement utilisée pour interpréter les données hydrochimiques [18-19]. Pour le traitement des données par l'analyse en composante principale, nous avons utilisé 12 variables (tableau 3) : la température, le $\mathrm{pH}$, la conductivité électrique, la turbidité les chlorures, les matières en suspension, l'oxygène dissous et les Matières Oxydables, la dureté totale, nitrate, nitrite et le sulfate et comme individus les 6 prélèvements effectués entre Janvier et Septembre 2016. Le tableau 3 montre les valeurs des paramètres physicochimiques (valeurs minimales, maximales et moyennes) des eaux brutes utilisées pour l'étude.

Tableau 3:-Les caractéristiques Statistiques descriptives des paramètres physico-chimique de l'eau brute

\begin{tabular}{|c|c|c|c|c|}
\hline & Minimum & Maximum & Moyenne & Ecart type \\
\hline $\mathrm{T}^{\circ}$ & 18.90 & 29.70 & 25.3 & 4.50233 \\
\hline $\mathrm{pH}$ & 7.10 & 7.90 & 7.3517 & 0.32423 \\
\hline Conductivité & 47.4 & 67.1 & 54.8833 & 6.83215 \\
\hline Turbidité & 21.30 & 330.00 & 103.7667 & 121.8012 \\
\hline $\mathrm{MO}$ & 0.92 & 3.44 & 2.0167 & 0.90714 \\
\hline $\mathrm{NO}^{-} 3$ & 0.75 & 5.55 & 2.4267 & 1.99702 \\
\hline $\mathrm{NO}{ }^{-} 2$ & 0.02 & 0.03 & 0.0145 & 0.00377 \\
\hline $\mathrm{TH}$ & 1.62 & 5.69 & 2.4667 & 1.56803 \\
\hline $\mathrm{Cl}$ & 2.01 & 17.24 & 8.3250 & 6.87941 \\
\hline
\end{tabular}




\begin{tabular}{|c|c|c|c|c|}
\hline MES & 9.02 & 275.00 & 84.8333 & 104.90586 \\
\hline O2 & 3.78 & 6.78 & 5.5900 & 1.38787 \\
\hline
\end{tabular}

L'étude statistique qui a été basée sur la matrice de corrélation intermédiaire entre les variables et l'analyse en composante principale est illustrée sur le tableau 4.

Tableau4:- Matrice de corrélations intermédiaires entre les variables.

\begin{tabular}{|c|c|c|c|c|c|c|c|c|c|c|c|}
\hline$\frac{\text { parame }}{\text { tre }}$ & $\underline{T^{\circ}}$ & $\underline{\mathrm{pH}}$ & Cond & Turb. & $\underline{\mathrm{MO}}$ & NO3- & NO2- & $\underline{\mathrm{TH}}$ & $\underline{\mathrm{MES}}$ & $\mathrm{Cl}^{-}$ & $\underline{\mathrm{O}} 2$ \\
\hline $\mathrm{T}^{\circ}$ & 1.000 & & & & & & & & & & \\
\hline $\mathrm{Ph}$ & -0.669 & 1.000 & & & & & & & & & \\
\hline Cond. & 0.037 & -0.092 & 1.000 & & & & & & & & \\
\hline Turb. & 0.491 & -0.613 & 0.666 & 1.000 & & & & & & & \\
\hline MO & 0.513 & -0.751 & 0.460 & 0.848 & 1.000 & & & & & & \\
\hline $\mathrm{NO}^{-}$ & 0.733 & -0.677 & $\begin{array}{l}- \\
0.130\end{array}$ & 0.611 & 0.498 & 1.000 & & & & & \\
\hline $\mathrm{NO} 2^{-}$ & -0.397 & -0.106 & 0.744 & 0.330 & 0.213 & $\overline{0} \mathbf{0} 379$ & 1.000 & & & & \\
\hline $\mathrm{TH}$ & 0.108 & -0.287 & $\overline{-}-583$ & 0.133 & 0.196 & 0.658 & $\overline{-} \mathbf{0 . 5 0 7}$ & 1.000 & & & \\
\hline MES & 0.450 & -0.638 & 0.629 & 0.995 & 0.856 & 0.624 & 0.345 & 0.197 & 1.000 & & \\
\hline $\mathrm{Cl}^{-}$ & -0.912 & 0.583 & $-\overline{0}-058$ & $-\overline{0}-602$ & $-\overline{0} \mathbf{-} 438$ & $-\overline{0} 892$ & 0.387 & $\overline{0}-292$ & $-\overline{0} 572$ & 1.000 & \\
\hline $\mathrm{O} 2$ & -0.654 & 0.761 & $-\bar{l}-163$ & $-\overline{0}-838$ & $-\overline{0} 739$ & $-\overline{0}-931$ & 0.085 & $-\overline{0.565}$ & $-\overline{0.856}$ & 0.803 & $\begin{array}{l}1.00 \\
0 \\
\end{array}$ \\
\hline
\end{tabular}

Les résultats de l'analyse statistique montrent des corrélations positives et significatives entre les paramètres physicochimiques tel que les MES, turbidité, MO, la température et la conductivité électrique et une autre corrélation positive et significative entre le $\mathrm{pH}$, chlorure et l'O2 dissous (tableau 5).

Tableau 5:- Discrimination des groupes de variations en fonction de leurs contributions sur les trois premières composantes principales

\begin{tabular}{|l|l|l|l|}
\hline Variable & Composante 1 & Composante 2 & Composante 3 \\
\hline O2 & -0.949 & 0.234 & -0.179 \\
\hline Turbidité & 0.932 & 0.308 & 0.102 \\
\hline MES & 0.929 & 0.287 & 0.191 \\
\hline MO & 0.838 & 0.181 & 0.214 \\
\hline NO3- & 0.821 & -0.529 & 0.027 \\
\hline Chlorure & -0.806 & 0.388 & 0.410 \\
\hline pH & -0.790 & 0.133 & -0.105 \\
\hline Température & 0.729 & -0.355 & -0.544 \\
\hline NO2 & 0.076 & 0.902 & 0.261 \\
\hline Conductivité & 0.409 & 0.885 & -0.144 \\
\hline TH & 0.289 & -0.726 & 0.600 \\
\hline
\end{tabular}

En prenant en compte la totalité des informations apportées par les trois composantes principales (Tableau 5), les différents éléments pris en compte dans l'analyse sont groupés en trois éléments.

Les groupes d'éléments les mieux définis par la composante principale 1 sont : O2, Turbidité, MES, MO, NO3 , Température, Chlorure et pH. Parmi ces variables, celles qui contribuent le plus à former la CP 1 on trouve deux groupes différents: Le premier regroupe Turbidité, MES, MO, vecteurs NO3 et la-Température, qui sont fortement corrélés entre elles et positivement à la F1, puisqu'ils définissent des propres de même direction et le deuxième groupe qui est rassemblé par le: $\mathrm{O} 2$, Chlorure, et $\mathrm{pH}$, qui sont corrélés négativement à la Composante1. 
Les groupes d'éléments les mieux définis par la composante principale $2: \mathrm{NO} 2$ et la Conductivité électrique représentent le groupe formé par deux éléments qui fortement corrélé à la composante principale 2.

Les groupes d'éléments les mieux définis par la composante principale 3 est le titre hydrotimétrique(TH).

\section{Rapport saisonnier:-}

Pour évaluer l'effet des changements saisonniers sur la qualité des eaux du site de prise d'eau d'AftoutEssahili on a calculé la moyenne de chaque paramètre pour chaque saison puis le rapport de la saison de pluie sur la saison sèche (figure 1).

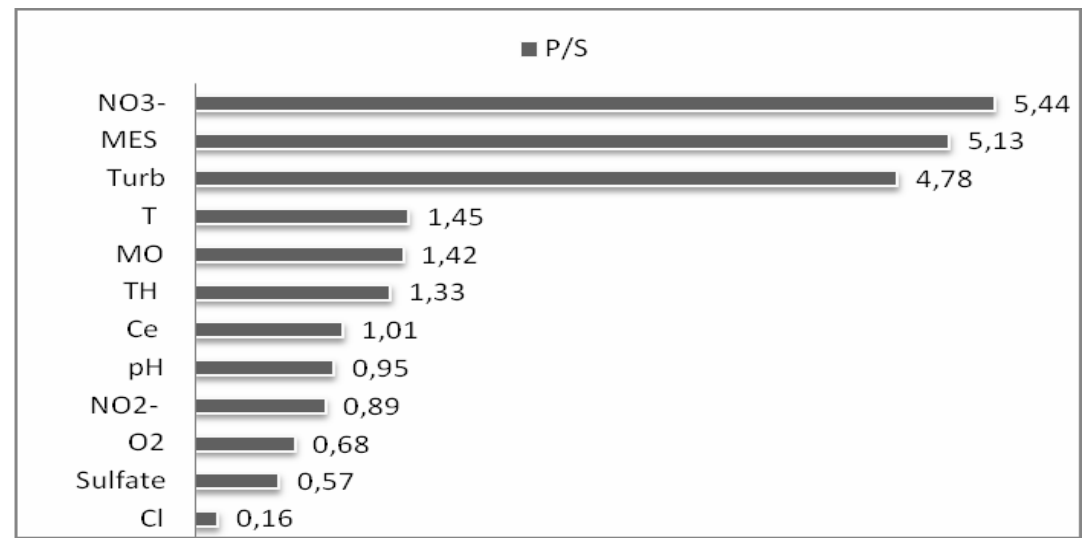

Figure 14:- Le rapport saisonnier (saison de pluie /saison sèche)

Le rapport saisonnier montre l'existence de deux groupes de paramètre:-

Groupe 1 : il s'agit des paramètres dont le rapport est supérieur à 1et même on peut diviser les paramètres de ce groupe à deux sous-groupe, les paramètres de variabilité élevée comme les NO3-, MES et la turbidité et celle de variabilité moyenne comme la $\mathrm{T}^{\circ}, \mathrm{MO}, \mathrm{TH}$ et la Conductivité électrique.

Groupe 2 : Comprend les paramètres de variabilité inférieure à 1, comme PH, NO2-, O2, et Chlorure.

\section{Discussions:-}

Les résultats obtenus durant cette étude ont permis de montrer que les eaux de surface présentent une turbidité et de MES variables au cours du temps. La turbidité maximale varie autour de 330 NTU ce qui est compatible avec la concentration maximale de MES de valeur maximale de $275 \mathrm{mg} . \mathrm{L}^{-1}$. Ceci montre que la technique de coagulation floculation utilisée pour la production d'eau potable pour la ville de NOUAKCHOTT sera fortement influencée par la variation de MES et de la turbidité. Par ailleurs la concentration des matières organiques admet comme valeur maximale de $3 \mathrm{mg} \cdot \mathrm{L}^{-1}$.

En effet, l'estuaire du fleuve Sénégal est situé dans le Sahel côtier zone avec une seule saison des pluies par an. Il a subi d'importants développements anthropiques depuis les années 1980 jusqu'en 1985 [17]. Il était une longue étendue d'eau, orienté nord-sud et séparés de la côte de l'Atlantique par un banc de sable

Les fleuves côtiers reçoivent souvent des apports d'eaux usées urbaines, en raison de la grande densité d'habitants dans les zones côtières domaines. En conséquence, ces rivières peuvent apporter de grandes quantités de nutriments et de polluants. L'enrichissement en nutriments conduit à l'eutrophisation d'un grand nombre de zones côtières Cloern zones avec une augmentation concomitante algale nuisible. Égouts, entre autres impacts, semble avoir un effet massif sur la santé humaine santé de la population par la contamination microbiologique des eaux et des coquillages côtiers récemment rapportées dans les études [20-21].

Aujourd'hui, l'état qualitatif des ressources en eau en Afrique est clairement dégradé par les pollutions d'origine domestique et agricole. En effet, la qualité d'une eau de surface est déterminée par sa teneur en organismes vivants et par les quantités de substances organiques et minérales qu'elle a entrainées. En outre, la qualité physicochimique des eaux de surface du fleuve Sénégal ne montre pas d'anomalies remarquables et respecte les conditions à tenir en compte pour préparer une eau potable. Toutefois, la concentration en MES dépasse les normes à respecter durant le 
mois d'Aout pendant la période pluviale $\left(275 \mathrm{mg} \cdot \mathrm{L}^{-1}\right)$. Ceci s'accompagne d'une augmentation de la turbidité (330 NTU au mois d'Aout). Dans ce cas la technique classique permettant de produire l'eau potable doit être bien optimisée et contrôlée afin d'aboutir à une eau qui respecte les normes de qualité. En effet, l'estuaire du fleuve Sénégal est loin d'être parmi les sites les plus contaminés en particulier en ce qui concerne des valeurs d'abondance (coliforme thermo tolérant (CTT) maximales [16]. Les mêmes auteurs ont rapporté que les variables les plus révélatrices de l'eutrophisation de l'estuaire étaient ceux liés aux communautés microbiennes. D'après les observations sur le terrain [16], l'eutrophisation de l'estuaire semble être le résultat des rejets des eaux usées.

D'après l'étude réalisée par Gisèle et al [17] sur le fleuve Sénégal les paramètres mesurés (nitrates, nitrites, température, $\mathrm{O}_{2}, \mathrm{PO}_{4} \ldots$ ) sur différents points de prélèvements varient avec le temps et avec le lieu de l'échantillonnage En raison de leur forte dépendance à l'égard des fluctuations de l'environnement les facteurs (température et salinité en particulier), les communautés de zooplancton sont de bons indicateurs de l'évolution des écosystèmes estuariens tropicale écosystèmes, qui varient considérablement dans le temps et dans l'espace [22-23].

Associés à d'autres éléments nutritifs comme les phosphates, l'excès de nitrates contribue à la dégradation des milieux naturels et notamment à l'Eutrophisation des eaux de surface (lacs, Oueds, étangs). A cause de leur transformation en nitrites dans l'organisme les nitrate, qui s'accumulent dans les eaux les rendre impropre à la consommation. Ils sont particulièrement dangereux pour les nourrissons et les fotus en raison du risque de méthémoglobinémie. Les nitrites peuvent aussi se combiner avec certains acides aminés, les constituants élémentaires des protéines, pour former des nitrosamines. Les stations de traitement de l'eau potable non équipés des processus de dénitrification qui rejettent dans les cours d'eau les nitrates issus de la dégradation de l'azote moléculaire et ammoniacal. En effet, les concentrations en nitrates et en phosphates ne présentent pas d'impacts négatifs sur la production d'eau potable vu que les teneurs de ces éléments restent très inférieurs à celles préconisées dans une eau utilisée pour la production d'eau potable.

Dans les variations à long terme du fleuve Sénégal, les périodes de ruissellement inférieures ont été identifiées montrant l'inégale distribution de la décharge de l'eau dans l'année [24]. En outre, l'élargissement du canal de banc de sable artificiel et sa transformation en un nouveau point de vente à la clôture de l'ancienne embouchure de la rivière a eu un effet important et rapide sur les échanges entre fleuve et l'océan [23]. Ceci influence fortement la qualité des eaux du fleuve sénégalais. Ceci pourra avoir un effet considérable sur la production d'eau potable au niveau de la station de production de l'eau potable au niveau de la ville de Nouakchott.

Les teneurs très élevées des eaux de surface en nitrates, matières phosphorées, matières organiques, engendrent de fortes nuisances environnementales, sanitaires et économiques : coûts élevés de production d'eau potable à usage domestique ou industriel, pollutions microbiologiques des eaux marines proliférations d'algues sur le littoral (marées vertes), etc. Ces phénomènes peuvent avoir des effets indirects graves en matière de développement économique : délocalisation d'entreprises agro-alimentaires, réduction de l'activité touristique, dégradation de l'image régionale et de celle de ses produits dans l'esprit des consommateurs...La lutte contre la pollution des eaux n'est pas un frein au développement économique mais constitue une aide à la décision pour aider les entreprises à bien gérer leurs effluents et trouver par la suite une place dans le marché. En agriculture, les nitrates et les phosphates sont des éléments nutritifs utilisés pour les plantes. Ils proviennent respectivement de l'azote et du phosphore et sont apportés aux plantes via les engrais chimiques, les déjections animales et parfois, les boues des stations d'épuration.

Les formes de pollutions sont multiples mais ont un point commun : elles aboutissent à la rivière et nuisent à la qualité de l'eau. Ainsi, une petite pollution, prise isolément, aura peu de conséquences sur la qualité des eaux. C'est la multiplication de ces rejets sur le bassin-versant qui nuira gravement à l'équilibre du fleuve Sénégalais récepteur. Cette dégradation, que l'on qualifie de diffuse, illustre bien le problème posé par la pollution d'origine industrielle puisque les effluents riches en métaux toxiques, en huiles, en solvants, de produits d'entretien ne sont pas traités avant rejet dans le milieu récepteur. L'utilisation massive des engrais et le drainage agricoles posent énormément de problèmes pour les pays en développement. En revanche, dans le cas des eaux de surface utilisé pour la production d'eau potable, les critères de prévention de la contamination sont calculés de façon à protéger un individu qui consommerait pendant toute sa vie une eau contaminée à cette concentration et des organismes aquatiques qui ont été accumulés la substance à partir de l'eau à la concentration des critères de prévention de la contamination. Les critères de prévention de la contamination peuvent aussi être basés sur des aspects 
organoleptiques ou esthétiques des substances pouvant altérer le goût, l'odeur ou la couleur de l'eau ou nuire aux travaux ménagers. L'utilisation d'une eau de mauvaise qualité esthétique peut amener le consommateur à modifier sa source d'eau pour une autre de meilleure apparence mais dont la qualité n'est pas garantie.

\section{Conclusion:-}

Les différents paramètres effectués sur les échantillons d'eau du fleuve Sénégal ont permis de dégager le comportement de certains paramètres descriptifs de la qualité physico-chimique des eaux. En effet, les analyses démontrent que la concentration de la quasi-totalité des paramètres répond aux normes en vigueur pour le choix d'une eau pour la production d'eau potable et, ne montre pas une pollution remarquable des eaux de surface. Toute fois les matières en suspensions atteignent $275 \mathrm{mg} / \mathrm{L}$ au mois d'Aout, avec une turbidité de 330NTU. Ceci influence fortement sur la qualité de l'eau prétraitée qui arrive à Nouakchott et dans ce cas, c'est la Station de potabilisation du PK 17 qui assure la production d'eau potable pour la ville de Nouakchott en intégrant parfois une phase de coagulation-floculation optimisée permettant d'aboutir à une eau potable respectant les normes de potabilité. La concentration en nitrate varie autour de $5 \mathrm{mg} / \mathrm{l}$ alors que la concentration en matières organiques marque une valeur de $3 \mathrm{mg} / \mathrm{l}$.

Sur la base des caractéristiques d'eau brute du fleuve Sénégal, les procédés de traitement appliquées doivent permettre la réduction des paramètres tel que la turbidité, les matières en suspension et les matières organiques.

\section{References:-}

1. Derwich E., Benaabidate L., Zian A., Sadki O., Belghity D., Larhyss Journal 8 (2010)101-112.

2. El Ouali L. A., Merzouki M., El Hillali O., Maniar S., Ibnsouda K. S., Larhyss Journal 9 (2011)55-72.

3. Rapport du Groupe de Banque Mondial. Sur l'état de l'environnement,(2013).

4. Kankou M.O.S.O., Thèse d'université, Université de limoges(2004).

5. Eby O. M., Afrique SCIENCE 04 (2008) $394-409$.

6. Degrement., Présentation du projet d'AEP de l'agglomération de Nouakchott à partir du fleuve Sénégal. (2008).

7. AFNOR (Association Française de Normalisation), la qualité de l'eau,(1999).

8. SNDE, Agence de Production de BeniNadji(2009).

9. OMS., rapport sur la santé dans le monde, l'état de la santé dans le monde.(1996).

10. Ghazali D., Zaid AN., Science Lib. Editions Mersenne 4 (2012)2111-4706.

11. Ward M.H., deKok T.M., Levallois P., Brender J., Gulis G., Nolan B.T., VanDerslice J., Environ. Health. Perspect. 113 (2005)1607-1614.

12. Powlson, D.S., Addiscott, T.M., Benjamin, N., Cassman, K.G., de Kok, T.M., van Grinsven, H., L'Hirondel, J.L., Avery, A.A. and van Kessel, C., J. Environ. Qual.37 (2008)291-295.

13. Arlot M. P., Thèse d'université, Université Paris 6, (1999)1-446.

14. Laurent F., Habilitation à Diriger des Recherches en Géographie UMR ESO, Université du Maine, (2012) 1-186.

15. Institut Bruxellois pour la gestion de l'environnement, Rapport sur l'état de la pollution Cadre général, 3-7 (2005).

16. TroussellierM.,GotP.,BouvyM.,MoboupM.,ArfiR.,LebihanF.,MonfortP.,CorbinD.,BernardC.,Marine Pollution Bulletin 48 (2004) 852-862.

17. Champalbert G., Pagano M., Arfi R. Chevalier C., Marine Pollution Bulletin 82 (2014)86-100.

18. Bennasser L., Thèse d'université, Université Ibn Tofaïl, Kenitra, (1997)1-177.

19. El Morhit M., Fekhaoui M., Serghini A., El belidi S., El abidi A .,Bennaakam R.,Yahyaoui A., JbilouM.,Bull.inst.sci. 30 (2008) 39-47.

20. Gesamp (IMO/FAO/UNESCO-IOC/WMO/WHO/IAEA/UN/UNEP Joint Group of Experts on the Scientific Aspects of Marine Environmental Protection) and Advisory Committee on Protection of the Sea, A sea of troubles. Rep. Stud. GESAMP, 70 (2001)35.

21. Schlacher T.A., Wooldridge T.H., Cah. Biol. Mar. 36 (1995)211-227.

22. Kibirige I., Perissinotto R., Estuar. Coast. Shelf Sc. 58 (2003)727-741.

23. Isupova M.V., Mikhailov V.N., Water Resour. 35 (2008)30-42.

24. Champalbert G., Pagano M., Sene P., Corbin D., Estuar. Coast. Shelf Sc. 74 (2007)381-394. 